\title{
Unsteady Blade Pressures on a Propfan: Predicted and Measured Compressibility Effects
}

M. Nallasamy

Sverdrup Technology, Inc.

Lewis Research Center Group

Brook Park, Ohio

July 1992

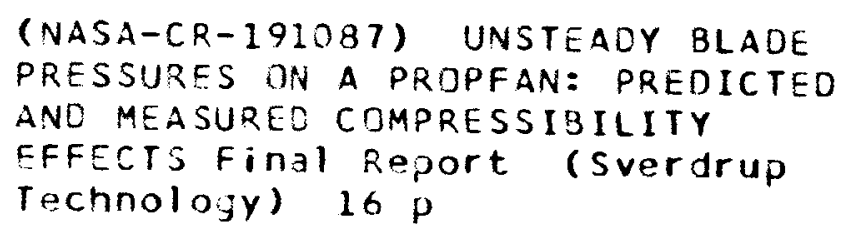

Prepared for

Lewis Research Center

Under Contract NAS3-25266

\section{NASก \\ National Aeronautics and \\ Space Administration}




\title{
UNSTEADY BLADE PRESSURES ON A PROPFAN: PREDICTED AND MEASURED COMPRESSIBILITY EFFECTS
}

\author{
M. Nallasamy \\ Sverdrup Technology, Inc. \\ NASA Lewis Research Center Group \\ Cleveland, Ohio
}

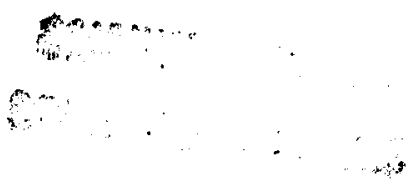

\begin{abstract}
$\underline{\text { Abstract }}$
The effect of compressibility on unsteady blade pressures is studied by solving the three-dimensional Euler equations. The operation of the eight-bladed SR7L propfan at $4.75^{\circ}$ angle of attack was considered. Euler solutions were obtained for three Mach numbers, $0.6,0.7$ and 0.8 and the predicted blade pressure waveforms were compared with flight data. The comparisons show that in general, the effect of Mach number on pressure waveforms are correctly predicted. The change in pressure waveforms are minimal when the Mach number is increased from 0.6 to 0.7 . Increasing the Mach number from 0.7 to 0.8 produces significant changes in predicted pressure levels. The predicted amplitudes, however, differ from measurements at some transducer locations. Also the predicted appearance of a shock in the highly loaded portion of the blade revolution is not indicated by the measurements. At all the three Mach numbers, the measured (installed propfan) pressure waveforms show a relative phase lag compared to the computed (propfan alone) waveforms due to installation effects. Measured waveforms in the blade tip region show nonlinear variations which are not captured by the present numerical procedure.
\end{abstract}

\section{Introduction}

The flow unsteadiness affects critically the propfan performance and near field noise levels. The unsteadiness may be the rasult of operation of the propfan at an angle-relative to the mean flow_direction or of distorted inflow caused by installation effects. Wind tunnel and flight tests were conducted on a 2.74 $\mathrm{m}(9 \mathrm{ft})$ diameter large-scale propfan to further understand the effects of flow unsteadiness ${ }^{1-3}$.

Unsteady blade pressure measurements of the large-scale propfan were first made in a transonic wind tunnel with angular inflow and (cylinder) wake inflow ${ }^{1}$. It was found that at takeoff conditions, high power cases resulted in the formation of a leading edge vortex on the blade. Then in the Propfan Test Assessment (PTA) program, flight tests were conducted to investigate the effect of inflow angle on the near-field noise level ${ }^{2}$. A sensitivity of about $1 \mathrm{~dB}$ per degree of

This Daper is ocenrea a work of the U.S. Governmem and is nol subjea to copynght protection in the United State. inflow angle change was found in flight measurements. Finally in the PTA follow-on flight tests, detailed unsteady blade pressure measurements ${ }^{3}$ were made on a specially designed instrumented blade. In this test, the blade suction surface had 20 pressure transducers distributed over three radial stations $(r / R=0.68$, 0.86 and 0.95 , where $r$ is the radial distance and $R$ is the blade tip radius) while the pressure surface had 10 pressure transducers distributed over two radial stations $(r / R=0.68$ and 0.95$)$. A nacelle tilt arrangement was employed to vary the inflow angle to the propfan.

Three sets of data were taken providing an unique database of detailed unsteady blade pressures in flight: 1. Low altitude $(580 \mathrm{~m})$, low speed (Mach number $\sim 0.3) 2$. High altitude $(10,500 \mathrm{~m})$, high speed (Mach number $\sim 0.8$ ) 3. A compressibility series in which the flight Mach number $(M)$ was varied (Mach number $\sim 0.6,0.7$ and 0.8 ), keeping the advance ratio and power coefficient (blade angle) constant. In each set, three nacelle tilt angles, $-3,-1$ (tilt down) and $2^{\circ}$ (tilt up), were considered giving an effective inflow angle variation of $5^{\circ}$. Efforts have been made to understand the unsteady flow features exhibited by these data and also to validate the prediction of three dimensional Euler analysis methods against the data.

The unsteady flow features of a propfan at takeoff (low speed, low altitude) were examined by Nallasamy ${ }^{4}$ by solving the three-dimensional Euler equations. It was found that the measured (installed propfan) blade pressure waveforms showed a relative phase lag compared to the computed (propfan alone) waveforms at the outboard $(r / R=0.95)$ station on both pressure and suction surfaces. However, on the pressure surface the magnitudes were found to be in good agreement with flight data at all inflow angles studied. On the suction surface, in addition to the relative phase lag, the measurements showed distortion of the waveforms. The extent of distortion increased with inflow angle.

Nallasamy and Groeneweg ${ }^{5}$ studied the unsteady flow features at cruise operating conditions. They showed that at inflow angles of 1.6 and 4.6 degrees 
passage shocks extending from suction to pressure surface formed and dissolved during each revolution of the blade. The computed unsteady blade pressures for $1.6^{\circ}$ were compared with wind tunnel data ${ }^{6}$. The comparisons showed good agreement of the predicted blade pressure waveforms with data at most of the transducer locations considered.

The effect of compressibility on steady blade pressures was examined ${ }^{7}$. It was found that with increasing Mach number, more significant changes occurred on the suction side than on the pressure side. The evidence of a compression wave started to develop when the Mach number reached 0.7. At a free stream Mach number of 0.78 , the compression wave fully developed into a trailing edge shock.

In the present paper, the effects of compressibility on unsteady blade pressures of a propfan are studied by solving the three dimensional Euler equations for angular inflow. The solutions are obtained for three Mach numbers, $0.6,0.7$ and 0.8 of the PTA follow-on compressibility test series, keeping advance ratio and blade angle constant. First, the predicted blade pressure waveforms for $M=0.8$ are compared directly with PTA follow-on flight data to assess the predictions at the high inflow angle, high speed case. Then the waveforms for three Mach numbers, 0.6, 0.7 and 0.8 , are compared with measured waveforms to evaluate the ability of the solution procedure in predicting the Mach number effects observed in flight tests. No effort is made here to quantify the effect of compressibility on propfan performance.

\section{Numerical Solution of Three-Dimensional Euler Equations}

The unsteady three-dimensional Euler equations governing the inviscid flow through a propfan are solved employing a solution procedure developed by Whitfield et al. ${ }^{8,9}$. In this procedure the Euler equations in conservative differential form are transformed from a Cartesian reference frame to a body fitted curvilinear reference frame. The transformed equations are then discretized employing a finite volume technique. A Lower-Upper (LU) implicit numerical scheme is used to solve the discretized equations. An approximate Riemann solver is used for block interface definitions. The flowfield is represented by a multi-block composite grid to limit the core memory requirements.

\section{Flow Configuration and Computational Grid}

The configuration considered is that of the eight bladed SR7L propfan of the flight test ${ }^{3}$. The direction of rotation of the propfan is clockwise, looking downstream. The azimuth angle, $\Phi$, is measured from ver- tical (top-dead-center) for aircraft installation as in the presentation of flight data in Ref.3 and increases in the direction of rotation (Fig. 0 ). The grid employed is an $\mathrm{H}$-grid with $107 \times 41 \times 25$ (axial by radial by circumferential) nodal points in each passage. The blade design hot coordinates are used to generate the grid. Each passage is divided into three blocks with $107 \times 41 \times 9$ grid points in each block. Thus 24 blocks of grid were used to describe the entire flowfield. Each blade surface is represented by $49 \times 27$ (axial by radial) grid points with higher resolution near the leading and trailing edges, the hub and the tip.

\section{Results and Discussion}

The unsteady three-dimensional Euler solutions were obtained for the following three flight test cases of Ref. 3 : (i) run $=144$, id $=1 \mathrm{c}$ and Mach number, $M=0.603$, (ii) run $=142$, id $=2 \mathrm{c}, M=0.701$ and (iii) run $=140$, id $=3 \mathrm{c}$ and $M=0.803$. These three runs had a nacelle tilt of $+2^{\circ}$. The effective inflow angle is dependent not only on the nacelle tilt angle but also on the airplane angle of attack and the upwash angle at the propfan (Fig. 0). For the above test runs the average value of the airplane angle of attack was $1.75^{\circ}$. The estimated upwash ${ }^{5}$ at the propfan was $1.0^{\circ}$. Thus the effective inflow angle to the propfan was $4.75^{\circ}$. The Euler solutions were obtained with an advance ratio of 3.17 , blade setting angle of $57^{\circ}$ and inflow angle of $4.75^{\circ}$ for all three Mach numbers.

The Euler solutions are obtained from an impulse start for three complete revolutions of the blade, to obtain a reasonably accurate solution. The results of the third revolution are analyzed and presented here. The predicted total power coefficient (for eight blades) are shown in Table $I$ along with the measured ones. In the experiments, the blade angle for $M=0.6$ is different from the other two. In the predictions, though the blade angle $\left(57^{\circ}\right)$ is maintained the same for all Mach numbers, the total power coefficient is under predicted by about $6 \%$ for $M=0.6$. The total power coefficients for $M=0.7$ and 0.8 are in reasonable agreement with data. The power per blade variation was found to show the expected sinusoidal variation due to inflow angle, in all three cases. The amplitude of the stabilized power coefficient during the third revolution varies $+109 \%$ and $-98 \%$ about the mean for $M=0.6,+120 \%$ and $-102 \%$ for $M=0.7$ and $+105 \%$ and $-104 \%$ for $M=0.8$.

First, the predicted and measured blade pressure waveforms are compared for the high Mach number case, $M=0.8$. Figure 1 shows the blade pressure waveforms at the transducer locations $(x / c$, where $x$ is the axial distance and $c$ is the blade chord) on the suction surface at the inboard radial station $r / R=0.68$. 
The predicted amplitudes agree fairly well with flight data. The measured waveforms show a relative phase lag compared to the predicted ones. This is in contrast to the takeoff case studied ${ }^{4}$ where such a phase lag was observed only at the outboard radial station. The observed phase lag seems to be due to installation effect, that is, the presence of the wing in flight tests as compared to the propfan alone configuration of the computation. Heidelberg and Woodward ${ }^{10}$ noted similar phase variations in their model tests in the wind tunnel with and without wing installation. In the present flight tests, the measured waveforms show a relative phase lag that varies from $17^{\circ}$ to $47^{\circ}$. The minimum lag, $17^{\circ}$, occurs at $x / c=0.4$ whereas near the leading edge, $x / c=0.05$, the lag is about $37^{\circ}$ and near the trailing edge, $x / c=0.8$, it is $47^{\circ}$.

On the pressure surface (Fig. 2) the predicted and measured waveforms differ significantly. This is due to the appearance of a computed shock wave during the highly loaded portion of the revolution. The measurements do not show such a shock wave. (Such a discrepancy was also observed in one of the steady (zero angle of attack) Euler computations reported ${ }^{7}$ ). The measured waveforms at $x / c=0.15$ and 0.8 show a phase lag compared to the predictions whereas at $x / c=0.4$ and 0.6 a phase lead is observed. A more detailed study is needed to establish if the appearance of the passage shock in the predictions is real and not an artifact of the numerical scheme employed.

Figure 3 shows the blade pressure waveforms at the transducer locations on the suction surface at the outboard radial station, $r / R=0.95$. Near the leading edge, the peak amplitude is underpredicted. The relative phase lag of the measured waveform compared to the computed ones exist at all transducer locations except that at $x / c=0.58$ and it is in the range of $34^{\circ}$ to $47^{\circ}$. At $x / c=0.58$ the measured waveform shows a small relative phase lead compared to the predictions.

The blade pressure waveforms on the pressure surface at the outboard radial station are shown in Fig. 4. At these transducer locations, the magnitudes are substantially overpredicted. Also, significant differences in shape of the waveforms are observed. However, a waveform of the shape similar to that predicted at $x / c=0.25$ has been observed in the wind tunnel tests of Heidelberg ${ }^{6}$ on the pressure surface for an inflow angle of $2^{\circ}$ (for example, see Fig. $5 \mathrm{c}$ in $\left.^{6}\right)$. However, it has not been possible to establish clearly, if the differences between the predicted and measured waveforms stem from the installation effects or not, due to lack of similar wind tunnel data at the high inflow angle considered here. At this outboard radial station, $r / R=0.95$, the value of the rel- ative phase lag of the measured waveform also varies widely.

Compressibility Effects: The effect of Mach number on unsteady blade pressure is demonstrated in Figs. 5 and 6 , in terms of blade surface pressure contours. Figure 5 shows the blade pressure at four azimuthal positions $\left(\Phi=0^{\circ}, 90^{\circ}, 180^{\circ}\right.$ and $\left.270^{\circ}\right)$ on the suction surface for all three Mach numbers. The blade pressure variation during a revolution is clearly seen to be significant at each Mach number, for the high inflow angle, $4.75^{\circ}$ considered here. The range of pressure variation during a revolution is of course maximum for $M=0.8$. Of particular interest is $\Phi=90^{\circ}$ position (in the highly loaded part of the revolution) where the area of low pressure region increases with increase in Mach number. Figure 6 shows the blade pressures at the same four azimuthal positions, as in Fig. 5, but on the pressure surface for three Mach numbers. It is seen that the cyclic variation of the blade pressure during a revolution is nearly the same for Mach numbers 0.6 and 0.7. But at Mach number $=0.8$, a shock appears (in the highly loaded region) at $\Phi=90^{\circ}$. The experimental data, however, do not show shock formation as discussed below.

Next, we present the predicted blade pressure waveform variations with Mach number along with the flight data. For clarity the predicted and measured data are presented side by side to show the compressibility effect in each case in all the succeeding figures. Figure 7 shows the predicted and measured waveforms at the transducer locations on the suction surface at $r / R=0.68$. First of all it is observed that the measured waveforms show a relative phase lag compared to the predicted ones at all the three Mach numbers. In general, the effect of increasing Mach number on the waveform is well predicted. Exceptions occur near the leading edge, $x / c=0.05$, where the maximum amplitudes are overpredicted for $M=0.6$ and 0.7 and at $x / c=0.8$ where a kink appears in the waveform for $M=0.8$.

The predicted and measured waveforms on the pressure surface at $r / R=0.68$ are shown in Fig.8 for three Mach numbers. The plots show that when the Mach number is increased from 0.6 to 0.7 very little change occurs in the waveforms, both in prediction and experiments. However, the magnitudes are overpredicted. For $M=0.8$, the predicted waveforms show sharp changes in magnitudes indicative of the presence of a shock (as shown in Fig. 6). But the experimental waveforms show that only near the leading edge, $x / c=0.15$, a noticeable change in waveform occurs when the Mach number is increased to 0.8. The relative phase lag of the measured waveforms persists 
at all Mach numbers.

For the outboard radial station, $r / R=0.95$, a comparison of predicted and measured waveforms is presented in Fig. 9. First, it is observed that the measured waveforms are distorted from sinusoidal form at all transducer locations for all Mach numbers. The distortion reduces with increase in Mach number. The tip vortex riding over the transducers for (highly loaded) part of the revolution may result in the measured shape of the waveform. The predictions show linear, sinusoidal behavior of the waveform at all transducer locations. The magnitudes are underpredicted. However, one trend is correctly predicted, that is, with increase in Mach number the response decreases at $x / c=0.25$ and 0.42 .

Euler solutions are known to predict qualitatively the formation of tip vortex ${ }^{11}$ in steady (zero angle of attack) flow, For angular inflow, the measurements seem to indicate that the spatial extent of the tip vortex on the blade (suction surface) depends on azimuthal position. During highly loaded part of the revolution, the tip vortex may extend over the transducer at $x / c=0.08,0.25,0.42$ and 0.58 . An adoptive grid which can track correctly the extent of the tip vortex and its strength at all azimuthal positions may be able to improve the predictions in the outboard region.

Figure 10 shows the pressure waveforms on the pressure surface at the outboard radial station, $r / R$ $=0.95$. The measured waveforms do not show much variation in absolute magnitude or shape with Mach number. That is true in predictions only for Mach numbers 0.6 and 0.7. Even at these Mach numbers the magnitudes are overpredicted. At $M=0.8$, the predictions show significant increase in response, which is not observed in experiments. In general at all Mach numbers, the maximum response occurs near the leading edge and the response reduces gradually towards the trailing edge, both in measurement and prediction. Significant relative phase lag of the measured waveform is observed at all transducer locations compared to the predicted ones.

\section{Concluding Remarks}

The effect of compressibility on unsteady blade pressures was studied by solving the threedimensional Euler equations. The unsteadiness is due to the operation of the propfan at $4.75^{\circ}$ angle of attack. Three Mach numbers, 0.6, 0.7 and 0.8 were considered and the predicted waveforms were compared with flight data.

Comparison of predicted and measured waveforms show that in general, the effect of Mach number on unsteady blade pressure waveform is correctly predicted. The changes in pressure waveforms are minimal when the Mach number is increased from 0.6 to 0.7 (except on the suction surface at $r / R=$ 0.95). Increasing the Mach number from 0.7 to 0.8 produces significant differences in predicted pressure levels. However, the predicted appearance of a shock at this Mach number is not indicated by the experiments. The measured waveforms show a relative phase lag compared to the predicted ones at most of the transducer locations. The phase lag is due to the installation effect, that is the installed propfan of the flight test compared to the propfan alone configuration of the computation.

The present numerical procedure is unable to reproduce the nonlinear variations of the measured waveforms. Perhaps an adoptive grid technique which captures accurately the extent and strength of the tip vortex may improve the prediction in the outboard region. When using the predicted (propfan alone) blade pressures to compute near-field acoustics for comparison with flight (installed) data, one has to phase shift the predicted pressure waveforms to correspond to installed measurement.

\section{Acknowledgement}

This work is sponsored by NASA Lewis Research Center under Contract NAS3-25266 with John F. Groeneweg as Project Manager.

\section{$\underline{\text { References }}$}

1. Bushnell, P., Gruber, M., and Parzych, D., "Measurement of Unsteady Blade Surface Pressures on a Single-Rotation Large-Scale Advanced Propfan with Angular and Wake Inflow at Mach Numbers from 0.02 to 0.70 ", NASA-CR-182123, Oct. 1988.

2. Little, B.H., Bartel, H.W., Reddy, N.N., Swift, G., Withers, C.C., and Brown, P.C., "Propfan Test Assessment (PTA) Flight Test Report", NASA-CR-182278, July 1989.

3. Parzych, D., Boyed, L.,Mesissner, W., and Wyrostek, A., "In Flight Measurement of Steady and Unsteady Blade Surface Pressure of a Single Rotation Large-Scale Advanced Propfan Installed on the PTA Aircraft", NASA-CR to be published.

4. Nallasamy, M., "Unsteady Blade Pressures on a Propfan at Takeoff: Euler Analysis and Flight Data", AIAA 92-0376, Jan. 1992.

5. Nallasamy and Groeneweg, J.F., "Unsteady Euler Analysis of the Flowfield of a Propfan at 
an Angle of Attack", Journal of Propulsion and Power, Vol. 8, no. 1, 1992, pp. 136-143.

6. Heidelberg, L.J., and Nallasamy, M., "Unsteady Blade Presures Measurements for the SR-7A Propeller at Cruise Conditions", AIAA 90-4022, Oct. 1990.

7. Nallasamy, M., Yamamoto, O., Wasrsi, S., and Bober, L.J., "Large-Scale Advanced Propeller Blade Pressure Distributions: Prediction and Data", Journal of Propulsion and Power, Vol. 7, no. 3,1991 , pp. $452-461$.

8. Whitfield, D.L., Swafford, I.W., Janus, J.M., Mulac, R.A., and Belk, D.M., "Three Dimensional Unsteady Euler Solutions for Propfans and Counter-Rotating Propfans", AIAA 87-1197, June 1987.

9. Janus, J.M., and Whitfield, D.L., "A Simple Time Accurate Turbomachinery Algorithm with Numerical Solutions of Uneven Blade Count Configuration", AIAA 89-0206, Jan. 1989.

10. Heidelberg, L.J., and Woodward, R.P., "Advanced Turboprop Wing Installation Effects Measured by Unsteady Blade Pressure and Noise", AIAA-87-2719, also NASA-TM-100200, Oct. 1987.

11. Nallasamy, M, Clark, B.J., and Groeneweg, J.J, "Euler Analysis of the Three-Dimensional Flow Field of a High-Speed Propeller: Boundary condition Effects, Journal of Turbomachinery, Vol. 109, 1987, pp. 332-339. 
Table I PTA Follow-on Test Cases ${ }^{3}$ :

Predicted and Measured Total

Power Coefficients

\begin{tabular}{|l|l|l|l|l|l|}
\hline Run & Mach & \multicolumn{2}{|l|}{ Blade } & Angle & \multicolumn{2}{l|}{ Power } & Coef. \\
\cline { 3 - 6 } No. & No. & Test & Euler & Test & Euler \\
\hline & & & & & \\
140 & 0.8 & 56.7 & 57.0 & 1.493 & 1.491 \\
142 & 0.7 & 56.6 & 57.0 & 1.533 & 1.549 \\
144 & 0.6 & 55.5 & 57.0 & 1.475 & 1.386 \\
\hline
\end{tabular}

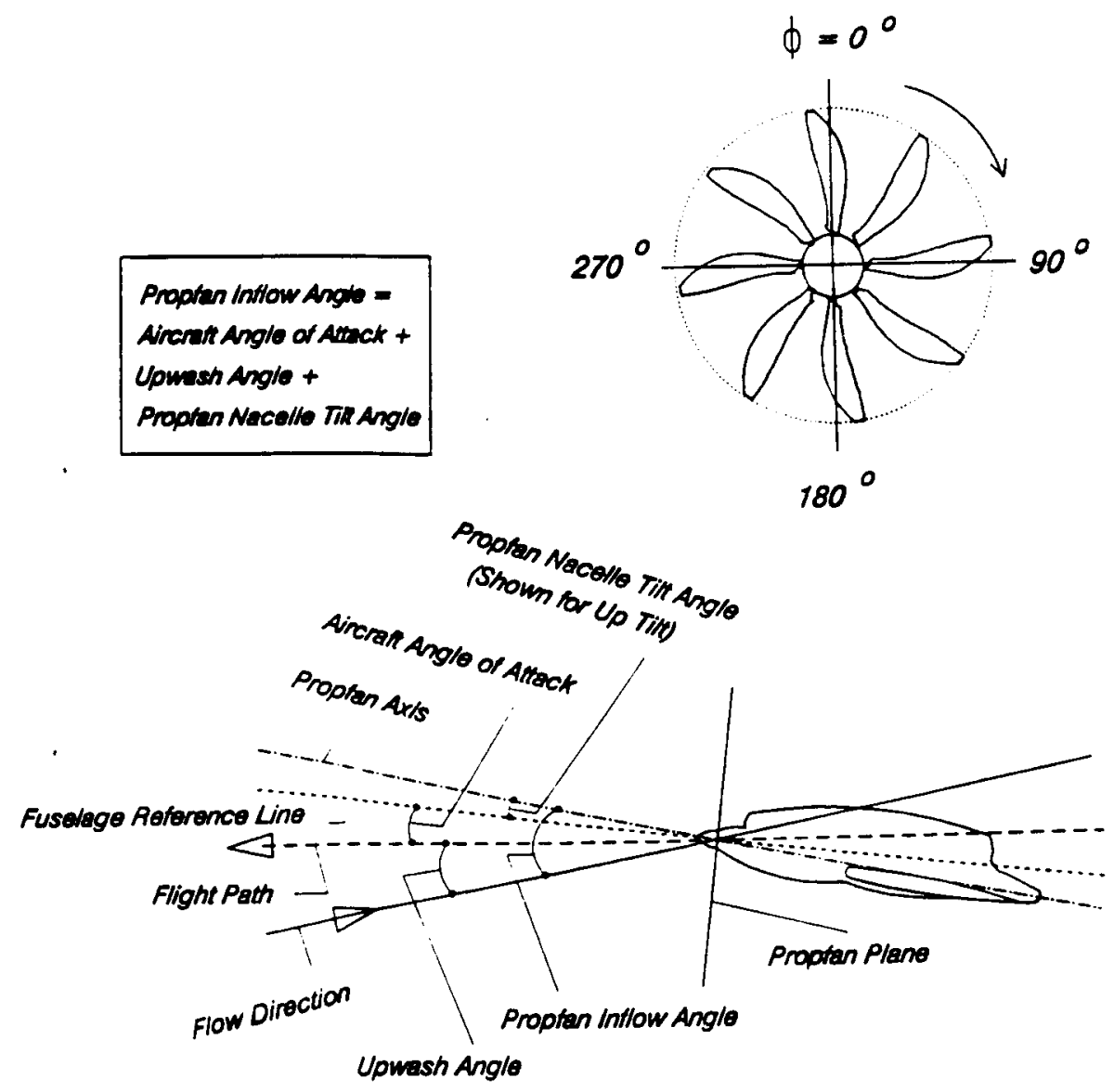

Figure 0. Definition Sketch 


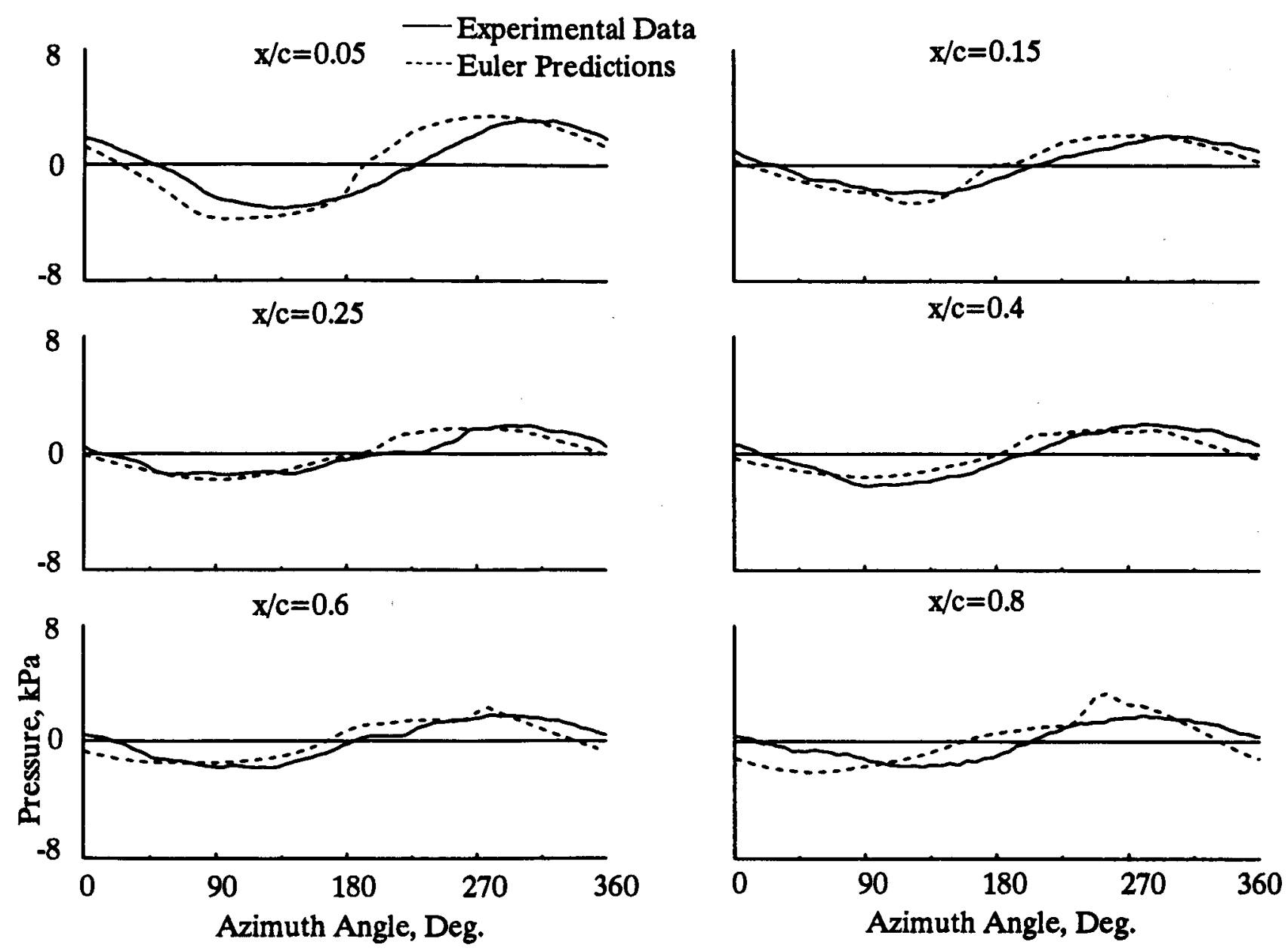

Fig.1 Pressure Waveforms on the Suction Surface at $r / R=0.68, M=0.8$
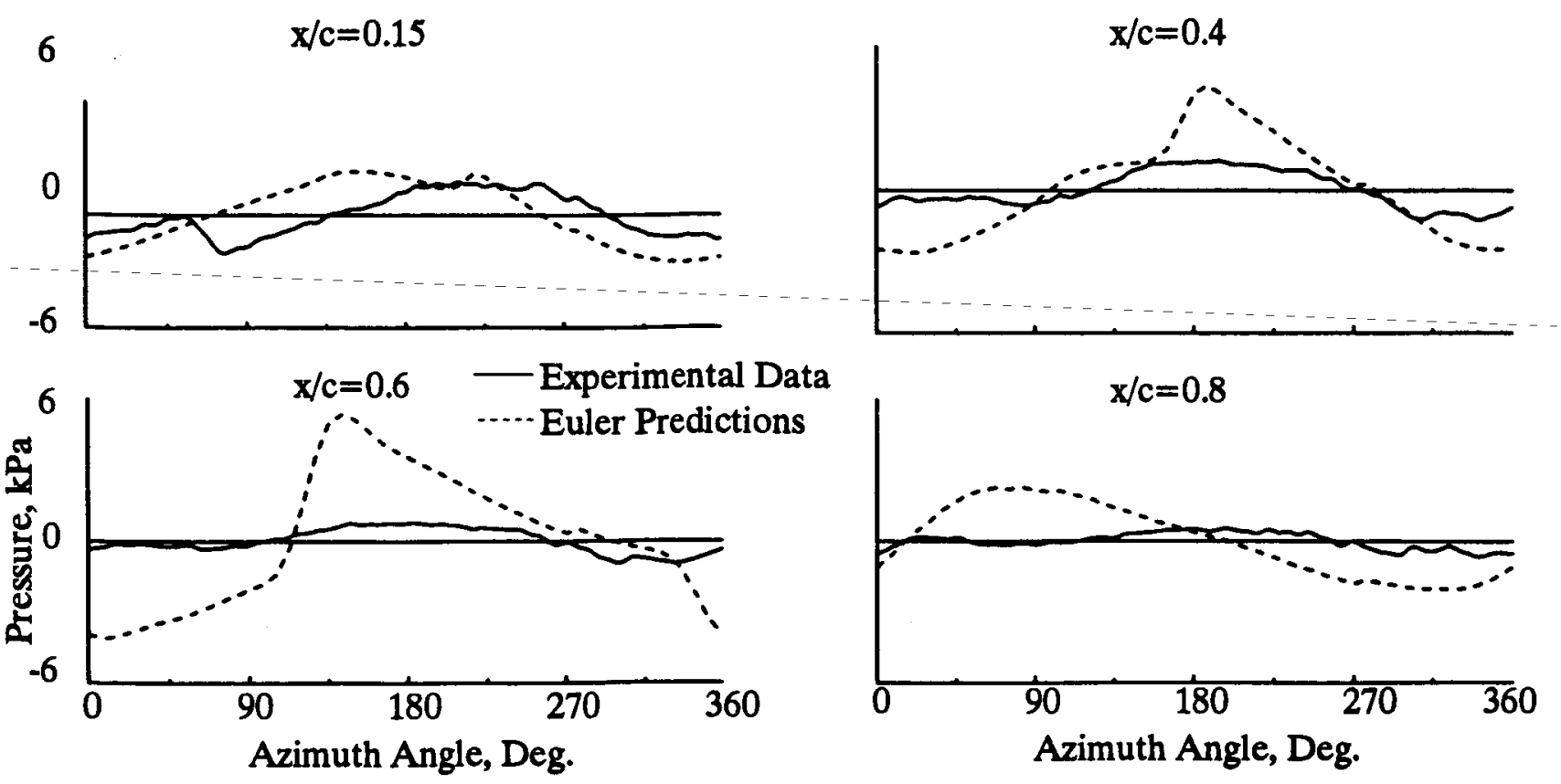

Fig.2 Pressure Waveforms on the Pressure Surface at $r / R=0.68, M=0.8$ 


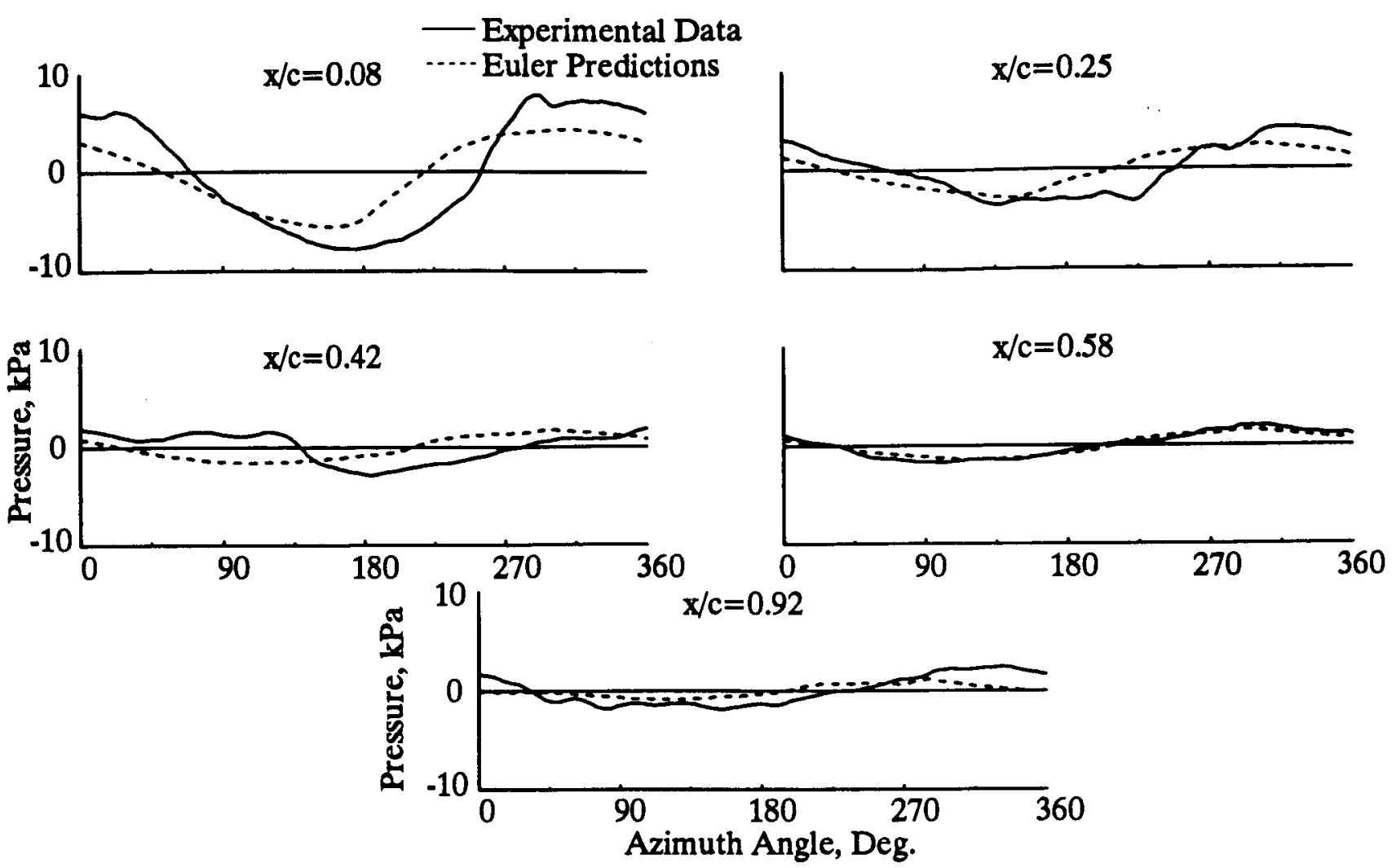

Fig. 3 Pressure Waveforms on the Suction Surface at $r / R=0.95, M=0.8$
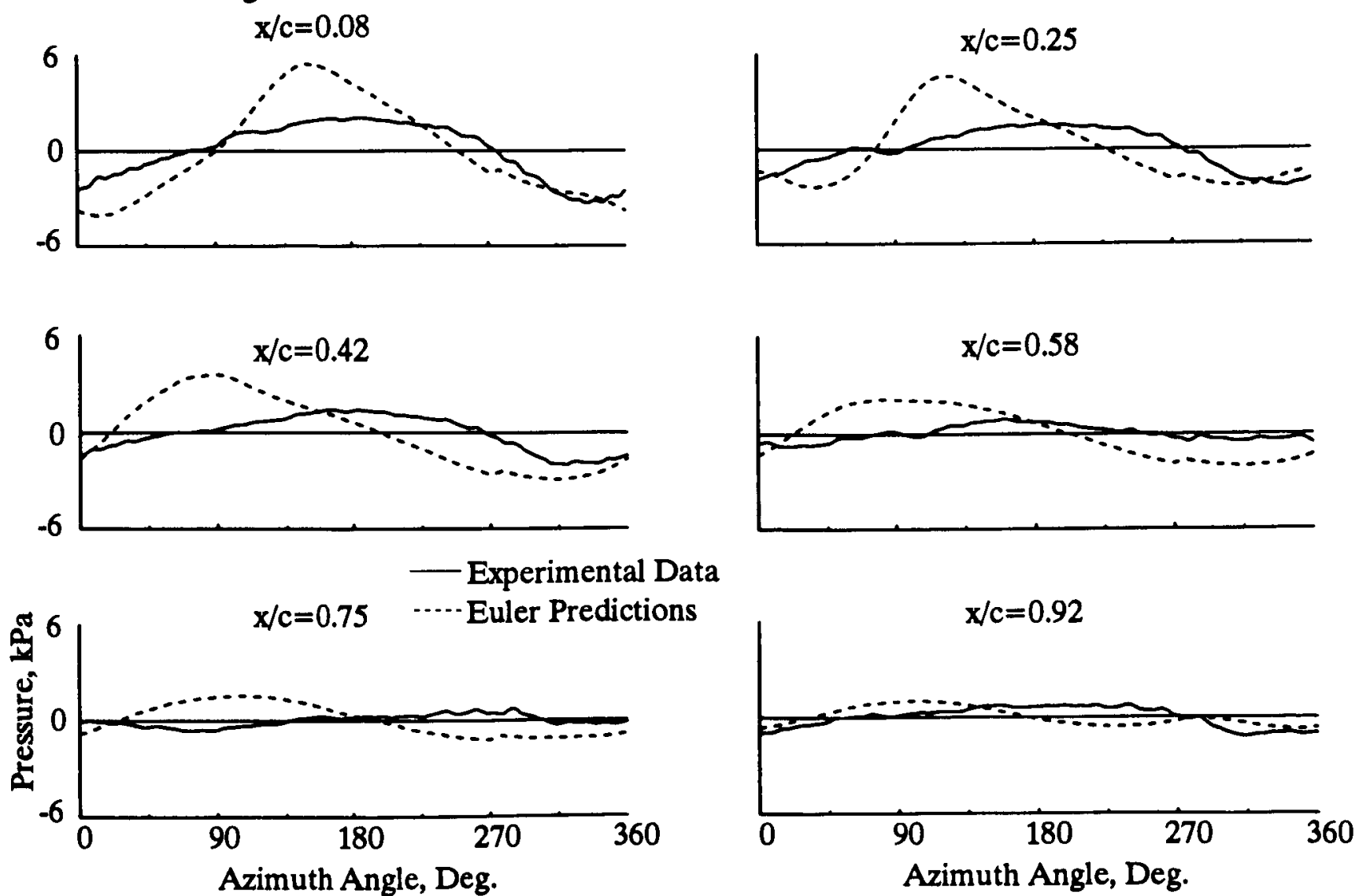

Fig. 4 Pressure Waveforms on the Pressure Surface at $r / R=0.95, M=0.8$ 

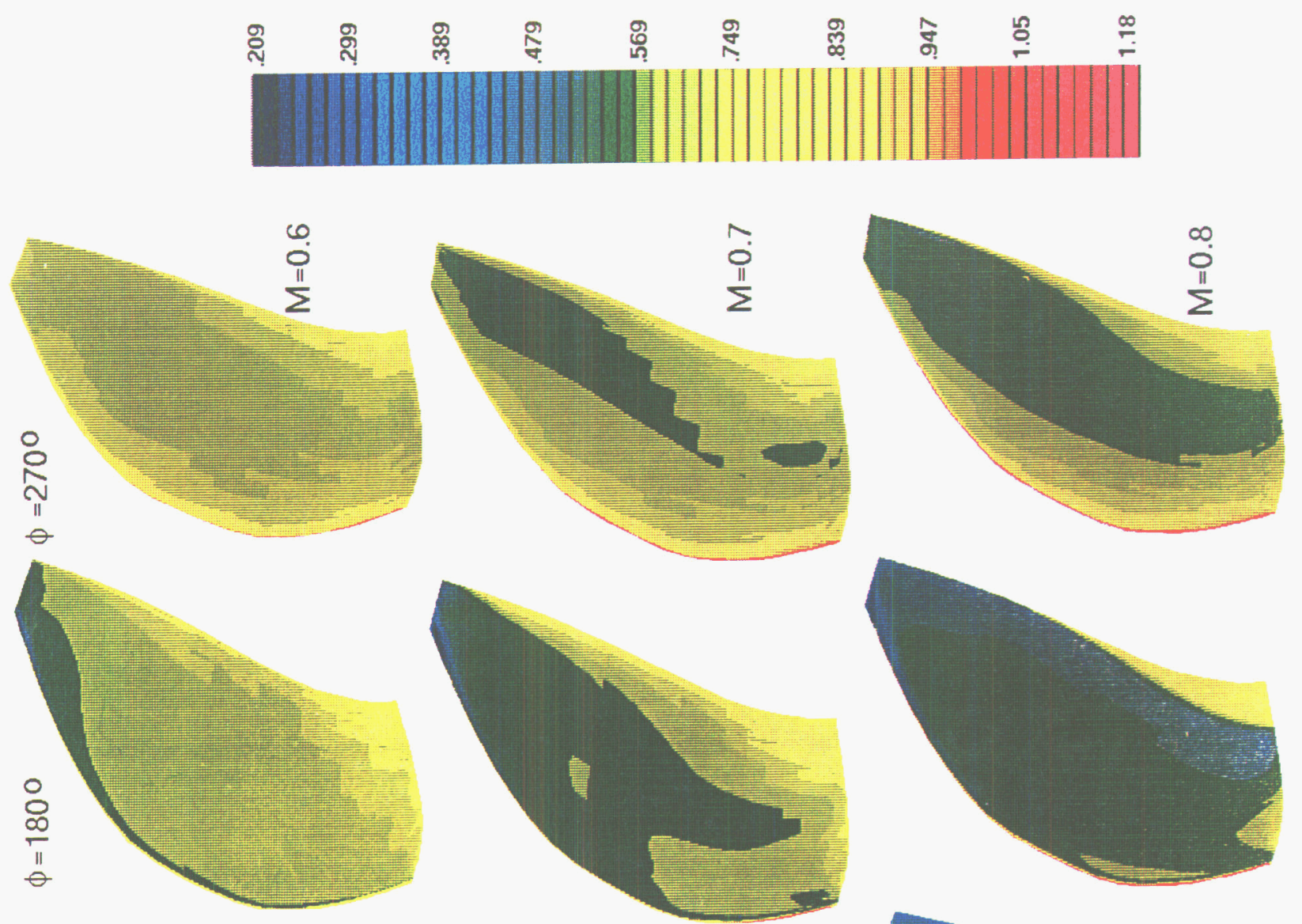

品
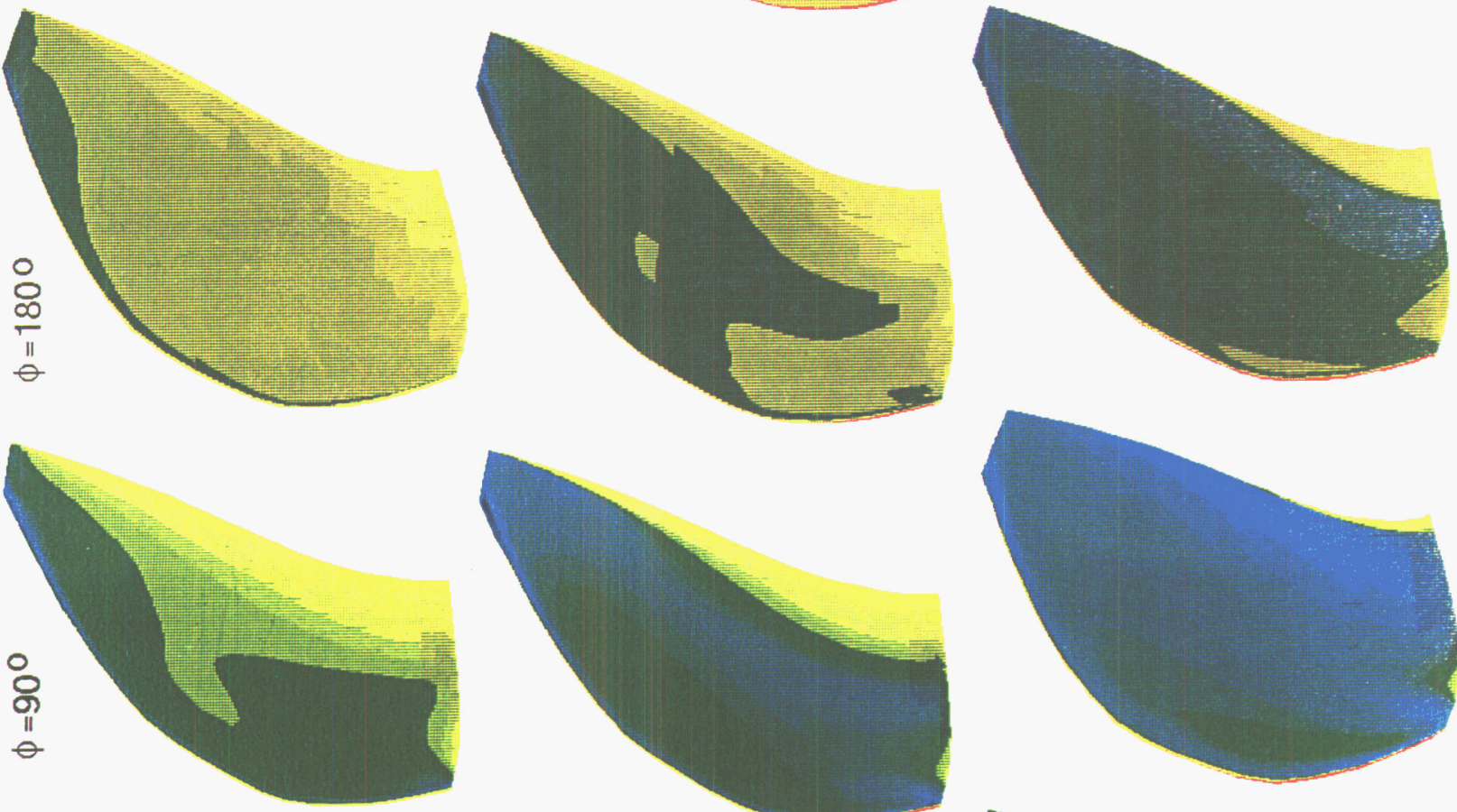

乒

등

웅
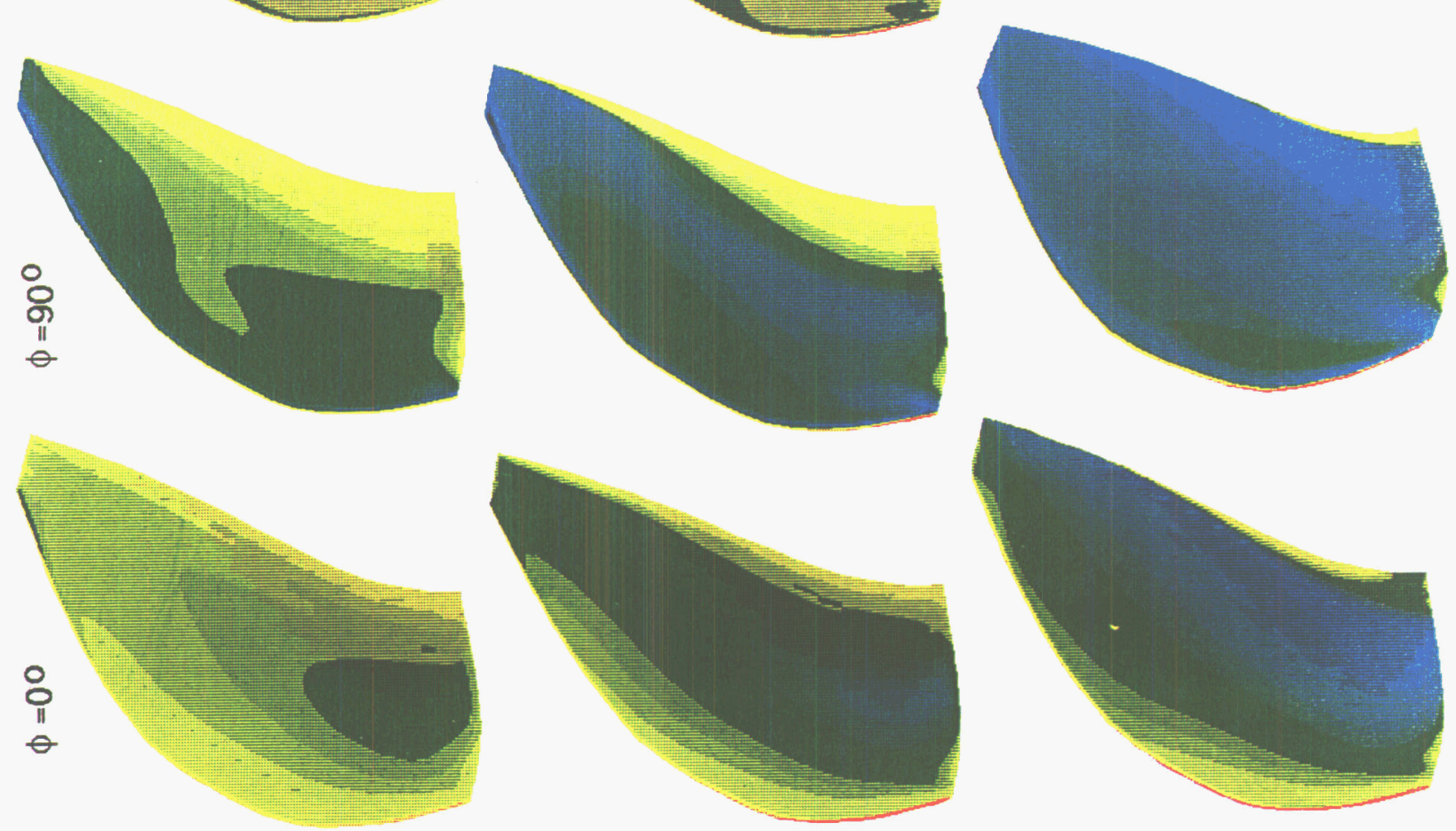


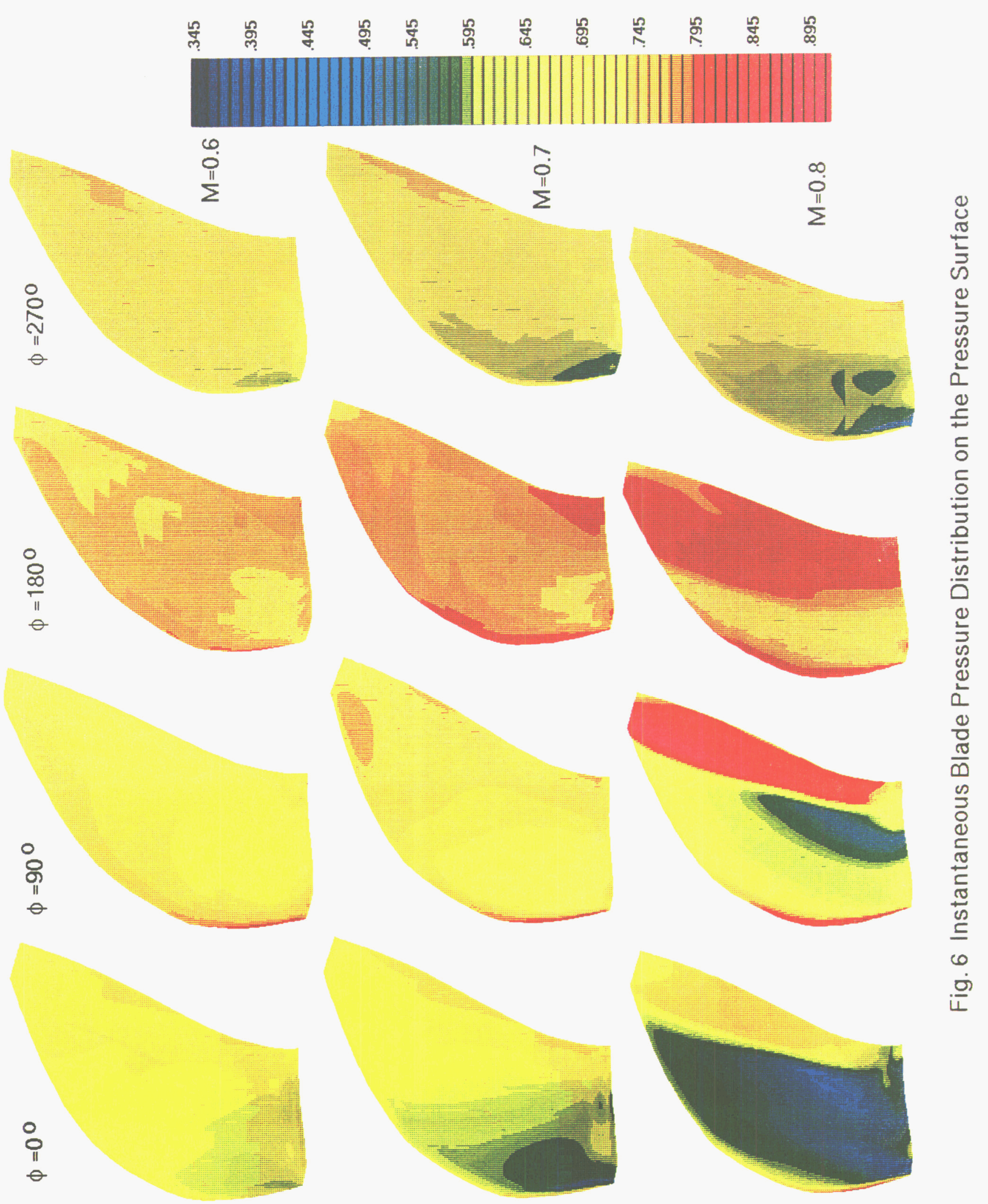




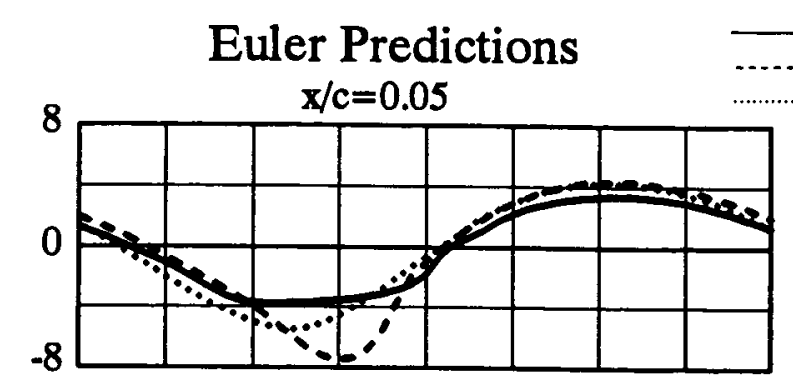

$\begin{aligned} M & =0.8 \\ \cdots \cdots . M & =0.7 \\ \cdots \cdots \cdots . . M & =0.6\end{aligned}$

Experimental Data
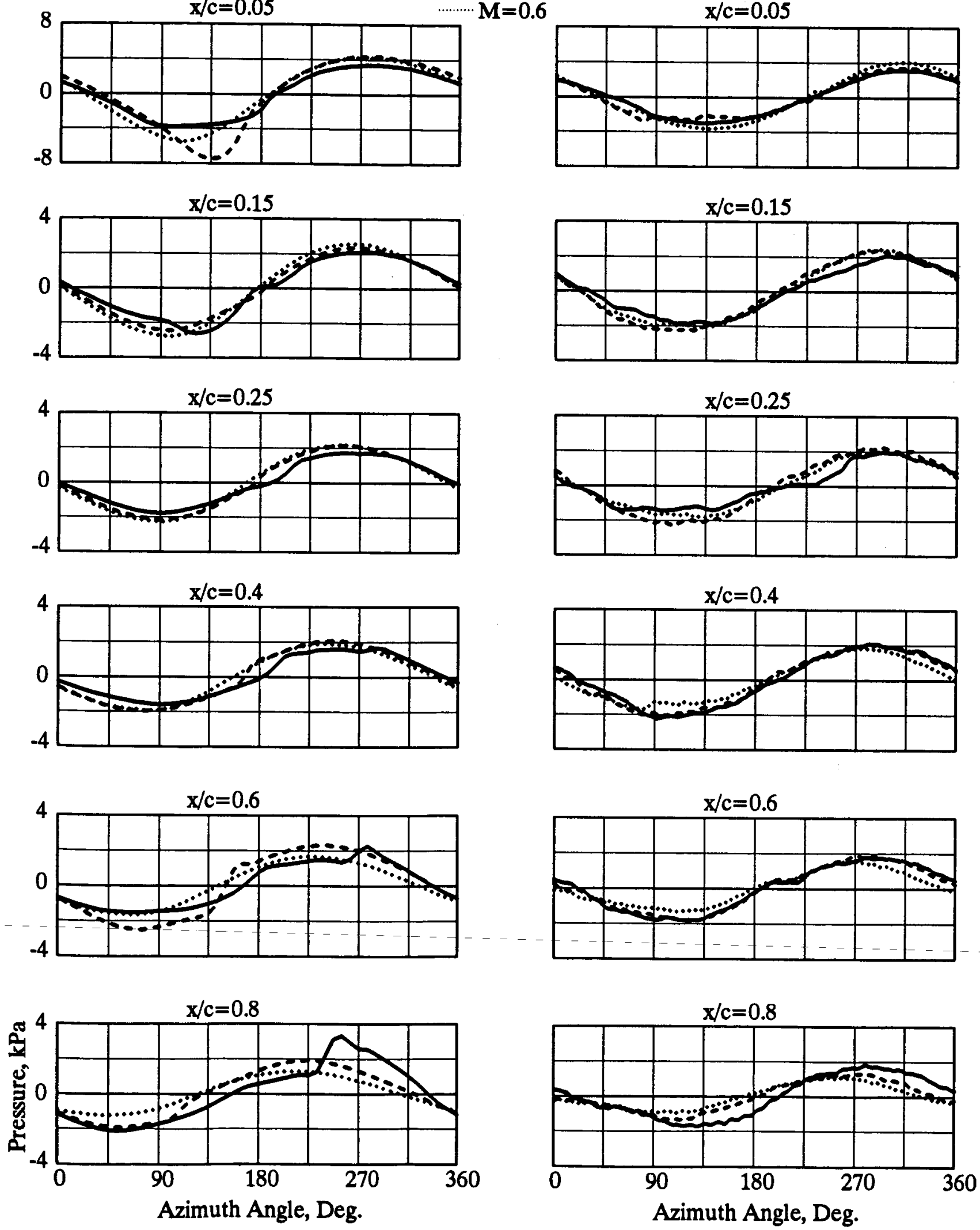

Fig. 7 Predicted and Measured Pressure Waveforms on the Suction Surface at $\mathrm{r} / \mathrm{R}=0.68$ 


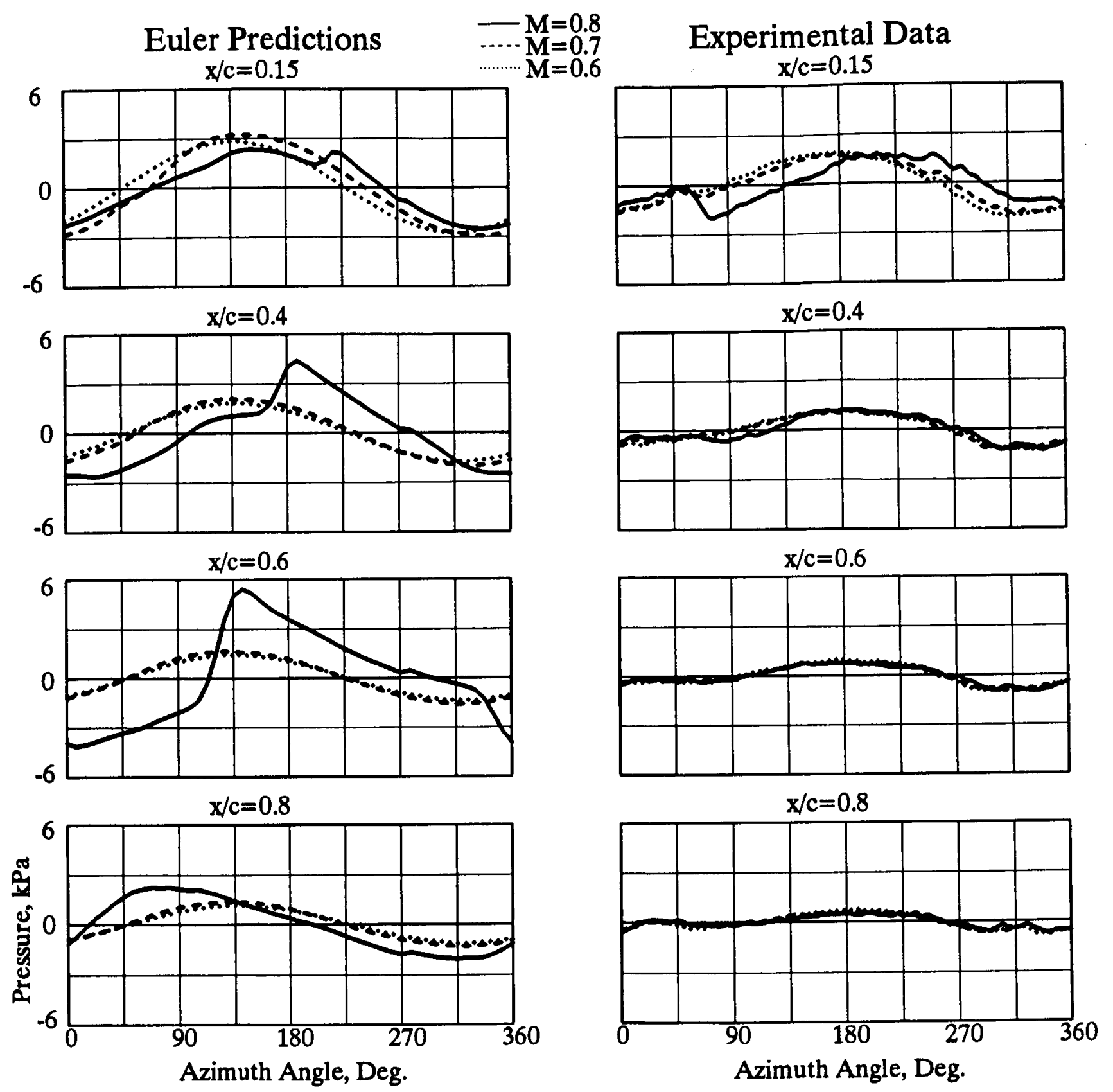

Fig. 8 Predicted and Measured Pressure Waveforms on the Pressure Surface at $\mathrm{r} / \mathrm{R}=0.68$ 


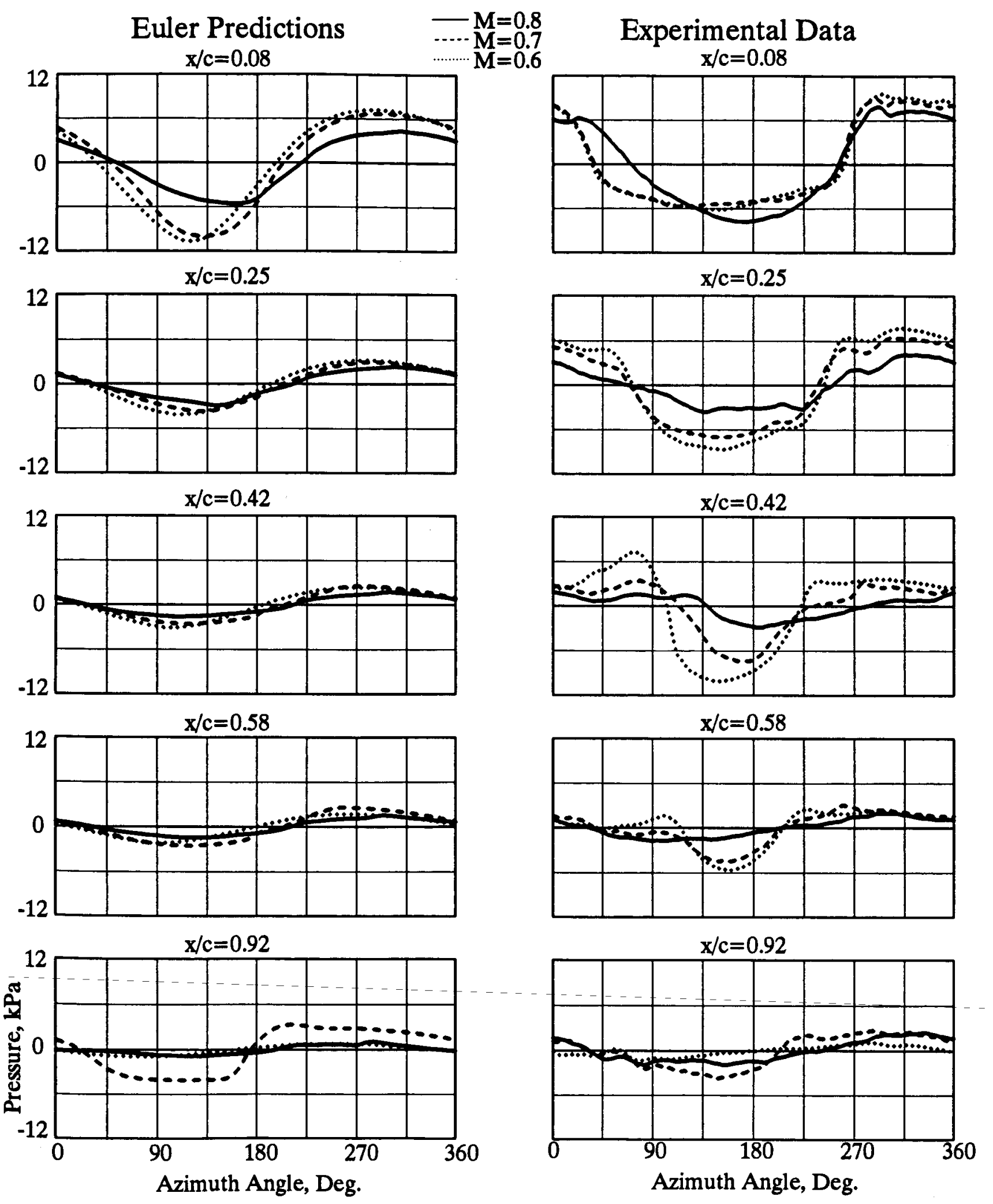

Fig. 9 Predicted and Measured Pressure Waveforms on the Suction Surface at $\mathrm{r} / \mathrm{R}=0.95$ 


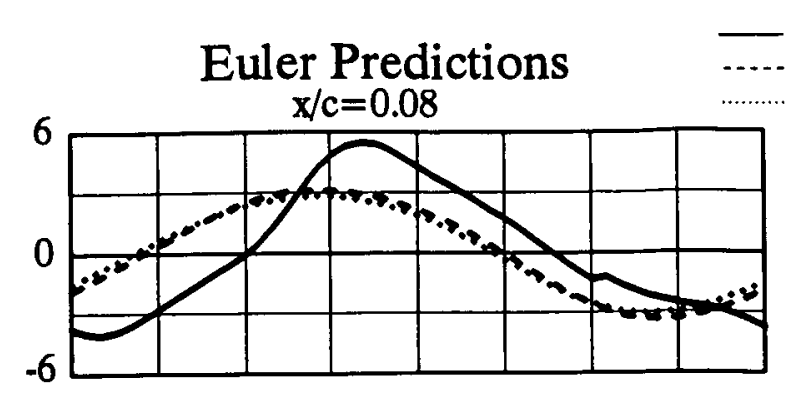

$M=0.8$
$M=0.7$

$\mathrm{M}=0.6$

Experimental Data $\mathrm{x} / \mathrm{c}=0.08$
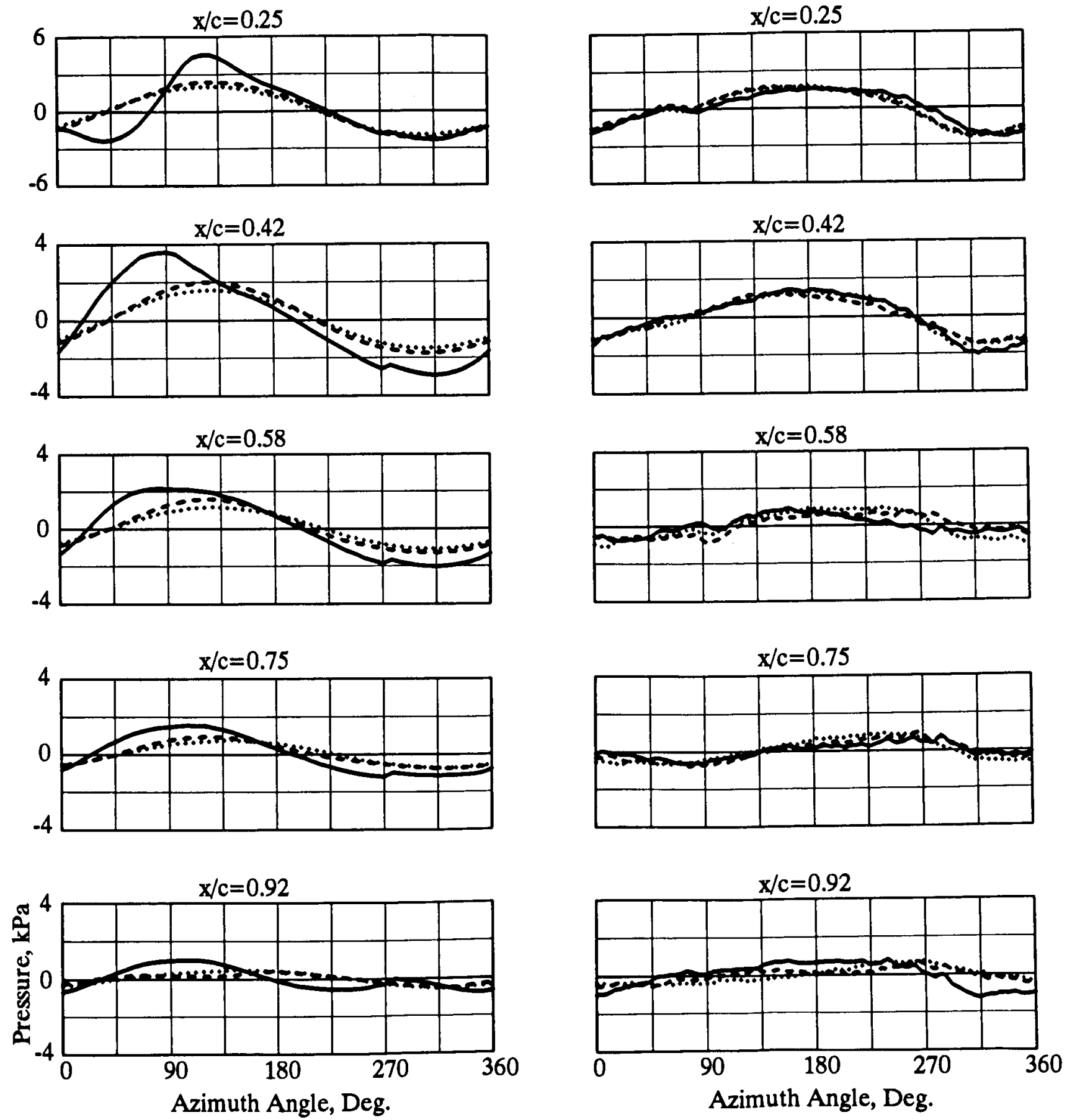

Fig. 10 Predicted and Measured Pressure Waveforms on the Pressure Surface at $\mathrm{r} / \mathrm{R}=0.95$ 
Public reporting burden for this collection of information is estimated to average 1 hour per response, including the time for reviewing instructions, searching existing data sources, gathering and maintaining the data needed, and completing and reviewing the collection of information. Send comments regarding this burden estimate or any other aspect of this collection of information, including suggestions for reducing this burden, to Washington Headquarters Services, Directorate tor information Operations and Reports, 1215 Jefferson Davis Highway, Suite 1204, Arlington, VA 22202-4302, and to the Office of Management and Budget, Paperwork Reduction Project (0704-0188), Washington, DC 20503.

\begin{tabular}{|l|l|l}
\hline 1. AGENCY USE ONLY (Leave blank) & $\begin{array}{c}\text { 2. REPORT DATE } \\
\text { July } 1992\end{array}$ & $\begin{array}{c}\text { 3. REPORT TYPE AND DATES COVERED } \\
\text { Final Contractor Report }\end{array}$
\end{tabular}

\section{TITLE AND SUBTITLE}

Unsteady Blade Pressures on a Propfan: Predicted and Measured

Compressibility Effects

WU-535-03-10

C-NAS3-25266

\section{AUTHOR(S)}

M. Nallasamy

\section{PERFORMING ORgANIZATION NAME(S) AND ADDRESS(ES)}

Sverdrup Technology, Inc.

Lewis Research Center Group

2001 Acrospace Parkway

Brook Park, Ohio 44142
8. PERFORMING ORGANIZATION REPORT NUMBER

E-7631

\section{SPONSORING/MONITORING AGENCY NAMES(S) AND ADDRESS(ES)}

National Aeronautics and Space Administration

Lewis Research Center

Cleveland, Ohio 44135-3191

10. SPONSORINGIMONITORING AGENCY REPORT NUMBER

NASA CR-191087

11. SUPPLEMENTARY NOTES

Prepared for the 28th Joint Propulsion Conference and Exhibit cosponsored by the AIAA, SAE, ASME, ASEE, Nashville, Tennessec, July 6-8, 1992. Project Manager, John F. Groeneweg, Propulsion Systems Division, (216) 433-3945.

12a. DISTAIBUTION/AVAILABILITY STATEMENT

12b. DISTRIBUTION CODE

Uncla'sified - Unlimited

Subject Categories 02 and 71

13. ABSTRACT (Maximum 200 words)

Trie effect of compressibility on unsteady blade pressures is studied by solving the three-dimensional Euler equations. The operation of the cight-bladed SR7L propfan at $4.75^{\circ}$ angle of attack was considered. Euler solutions were obtained for three Mach numbers, 0.6, 0.7 and 0.8 and the predicted blade pressure waveforms were compared with flight data. The comparisons show that in general, the effect of Mach number on pressure waveforms are correctly predicted. The change in pressure waveforms are minimal when the Mach number is increased from 0.6 to 0. . Increasing the Mach number from 0.7 to 0.8 produces significant changes in predicted pressure levels. The predicted amplitudes, however, differ from measurements at some transducer locations. Also the predicted appearance of a shock in the highly loaded portion of the blade revolution is not indicated by the measurements. At all the three Mach numbers, the measured (installed propfan) pressure waveforms show a relative phase lag compared to the computed (propfan $:$ !one) waveforms due to installation effects. Measured waveforms in the blade tip region show nonlinear variations which are not captured by the present numerical procedure.

\section{SUBJECT TERMS}

Unsteady aerodynamics; Euler analysis; Propfan acrodynamics; Acroacoustics of propfans

\begin{tabular}{|c|c|c|}
\hline $\begin{array}{c}\text { 17. SECURITY CLASSIFICATION } \\
\text { OF REPORT } \\
\text { Unclassified }\end{array}$ & $\begin{array}{c}\text { 18. SECURITY CLASSIFICATION } \\
\text { OF THIS PAGE } \\
\text { Unclassified }\end{array}$ & $\begin{array}{c}\text { 19. SECURITY CLASSIFICATION } \\
\text { OF ABSTRACT } \\
\text { Unclassified }\end{array}$ \\
\hline
\end{tabular}

,

